\title{
Effect of Different Exercise Mode and Ursolic Acid Supplemen- tation on FNDC5 and UCP1 Gene Expression and Plasma Irisin in Rats
}

\author{
Mahzad Teimourian ${ }^{1}$, Hoseyn Fatolahi ${ }^{2 *}$ (D) and Hasan Mateenhomaei ${ }^{1}$ \\ ${ }^{1}$ Department of Exercise Physiology, Central Tehran Branch, Islamic Azad University, Iran \\ ${ }^{2}$ Department of Physical Education, Pardis Branch, Islamic Azad University, Iran
}

*Corresponding author: Hoseyn Fatolahi, Department of Physical Education, Pardis Branch, Islamic Azad University, Pardis, Iran

\begin{abstract}
Background: Each exercise mode can lead to a specific effect. On the other hand, herbal supplements increase the effects of exercise. Yet the interactive effect of exercise mode and Ursolic Acid (UA) on the Brown Adipose Tissue (BAT) has not been well explored. The aim of the present study was to investigate the effect of 12-week Aerobic Training (AT), Resistance Training (RT), and High Intensity Interval Training (HIIT) along with UA supplementation on muscle FNDC5 and adipose tissue UCP1 gene expression as well as plasma irisin levels.
\end{abstract}

Methods: A total of 40 Male Wistar rats were randomly divided into 4 groups, including: (1) Control (C), (2) AT+UA, (3) RT+UA, and (4) HIIT+UA. UA (200 mg/kg) was injected intraperitoneally twice a day for 1 week. The training protocol included 12 weeks ( 5 sessions per week). Intensity of RT was applied from 50\% to 100\% 1-RM. Also, HIIT (1 min at $90-100 \%, 60-66 \mathrm{~m} / \mathrm{min}$ and $2 \mathrm{~min}$ at $50-60 \%, 18-22 \mathrm{~m} /$ $\mathrm{min}$ ) and AT (50-60\%) were carried out in the form of running on the treadmill.

Results: FNDC5 ( $F=23.444, P=0.001, \eta=0.304)$, and UCP1 gene expression ( $F=20.315, P=0.001, \eta=0.260)$ and plasma irisin levels $(F=9.207, P=0.001, \eta=0.249)$ were significantly different among the studied groups. The effect of AT $(p=0.01)$ was more than RT $(p=0.03)$ and HIIT $(p=0.02)$.

Conclusion: Regarding the changes observed in the present study, the interactive effect of AT and UA supplementation may be more on brown adipose tissue production. Also, based on the specificity principle of training, it is likely that each variable can be influenced by a unique exercise mode.

\section{Keywords}

Exercise mode, Ursolic acid, Brown adipose tissue, Irisin

\section{Introduction}

The effect of regular physical activity on controlling and improving the disease and physiological capacities has been confirmed in previous studies. However, it is necessary to study on the physiological mechanisms of trainings and prescribing different exercise modes [1]. Aerobic and resistance trainings are more commonly known, and extensive studies have been done on these types of training [1]. However, there is a need for further research on a new exercise training method called high intensity interval training (HIIT) [2-4]. In fact, the specificity principle of training for each of the variables in the field of exercise physiology should be studied separately.

One of the important issues in this area is the study of adipose tissue changes. The formation of brown adipose tissue is one of the important physiological signaling pathways. Brown Adipose Tissue (BAT) prevents the incidence of metabolic syndrome [5]. FNDC5 (Fibronectin type III Domain-Containing Protein 5) gene expression [6] and irisin secretion with muscular origin [7] also interfere with this effect. Non-shivering thermogenesis is the most important function of BAT. This function is regulated by UCP1 (Uncoupling Protein) -also known as thermogenin- located in the mitochondrial membrane. The signaling pathway for transformation of white adipose to BAT is influenced by physical activity involving the associations between irisin, FNDC5, and UCP1 gene expression $[6,7]$.

Citation: Teimourian M, Fatolahi H, Mateenhomaei H (2020) Effect of Different Exercise Mode and Ursolic Acid Supplementation on FNDC5 and UCP1 Gene Expression and Plasma Irisin in Rats. Int J Sports Exerc Med 6:160. doi.org/10.23937/2469-5718/1510160

Accepted: February 20, 2020; Published: February 22, 2020

Copyright: (C) 2020 Teimourian M, et al. This is an open-access article distributed under the terms of the Creative Commons Attribution License, which permits unrestricted use, distribution, and reproduction in any medium, provided the original author and source are credited. 
Irisin has been shown to have a positive relationship with the metabolic state, blood pressure, and endothelial cell function. In addition, irisin is effective in controlling the obesity and insulin resistance in men and women. The increase in BAT produced through irisin can play a role in controlling the metabolic syndromes, and its role in thermogenesis is important $[5,8]$. Despite the role of BAT, the pharmacologic signaling pathways influencing the growth of this tissue are still unknown [8]. Regular physical activity has also led to the conversion of adipose tissue into various species, although limited human studies are available in this regard $[9,10]$. Exercise training has been reported as an essential factor in the formation of BAT on the FNDC5 and irisin signaling pathways activit [10-12].

In addition, the effect of training on each of the physiological variables can be increased through the supplementation of plant or chemical materials. The use of Ursolic Acid (UA) supplement is one of the effective factors in increasing the BAT [13]. The apple peels and many other herbal compounds contain UA [14]. The UA has a broad pharmacological effect including anti-tumor, anti-inflammatory, anti-oxidant, and anti-allergic properties $[15,16]$. In addition, the role of UA in controlling the obesity, dyslipidemia, and atherosclerosis has been identified [17]. The UA increases the skeletal muscle and BAT, and is effective in controlling the obesity by improving the glucose intolerance and fatty liver disease [18]. This effect is improved through collaborative pathways such as irisin [19]. The UA has been investigated in relation to the field of exercise physiology, and has been shown to reduce muscle injuries in trained men [20]. Since one bout of intense exercise can cause oxidative stress, therefore, administration of anti-oxidant and anti-inflammatory supplementation along with physical activity is essential.

Briefly, regular physical activity has been shown to increase the BAT. Nevertheless, there is still debate as to what type of physical activity can increase this effect. On the other hand, some researchers are looking for the supplements to use along with training to develop the effects of exercises. The UA is one of these materials, whose effect on the BAT needs to be investigated. The UA forms the BAT by influencing the signaling pathways. It has been shown that, the stimulation of FNDC5 and UCP1 expression and irisin secretion are the most important pathways for BAT alteration. However, the simultaneous effect of the exercise mode and the UA supplementation has not been studied. Based on the literature reviewed, it is assumed that, different exercise modes can affect changes in the white-to-brown adipose tissue conversion signaling pathways. Therefore, the aim of present study was to evaluate the effect of HIIT, aerobic, resistance training along with UA supplementation on the muscle tissue FNDC5 and adipose tis- sue UCP1 gene expression and plasma irisin concentration in male Wistar rats.

\section{Methods and Materials}

\section{Permissions}

The ethics committee of Islamic Azad University, Central Tehran Branch initially approved the experimental procedures and study protocols (No. 10121436962031). This study was conducted in accordance with the NIH (National Institutes of Health Guide for the Care and Use of Laboratory Animals: No. 80-23), which emphasized the use of minimal animal sacrificed and minimal pain during the study.

\section{Animals and studied groups}

A total of 40 male Wistar rats (aged 10 weeks) were randomly and equally divided into 4 groups including: (1) Control (C) $\left(\mathrm{W}_{(\mathrm{g})}=280.25 \pm 10.88\right)$, (2) Aerobic training + ursolic acid (AT+UA) $\left(W_{(g)}=257.25 \pm 11.89\right)$, (3) Resistance training + ursolic acid $(\mathrm{RT}+\mathrm{UA})\left(\mathrm{W}_{(\mathrm{g})}=276.87 \pm\right.$ 22.30) and (4) HIIT + ursolic acid (HIIT+UA) (W $\mathrm{W}_{(\mathrm{g})}=262.87$ \pm 17.49 ). Ursolic acid supplement (Enzo Life Sciences) (200 mg/kg in corn oil solution) was injected intraperitoneally twice a day for 1 week [18]. Animals were kept under controlled light/dark conditions (12-hour cycle of light and dark, light period started at 6:00 am and darkness period started at $6: 00 \mathrm{pm})$, temperature $(22 \pm 3$ ${ }^{\circ} \mathrm{C}$ ), and humidity (32\%). Animals had free access to the standard water and food (Harlan Teklad formula 7013).

\section{Training methods}

All training methods were performed during 12 weeks ( 5 sessions per week). Duration of all exercises was the same (approximately 30 minutes for each exercise). Resistance training included climbing a ladder (110 cm height, $80 \%$ slope and $2 \mathrm{~cm}$ ladder distance) in the form of 3 periods with 6 repetitions. The rest duration between the periods was 3 minutes and the rest interval between repetitions was 45 seconds. The last weight the animal was able to carry up was considered $1 \mathrm{RM}$ and the first training session started with $50 \%$ of 1-RM. Resistance was gradually increased from $50 \%$ to 100\% 1-RM [21].

HIIT included running on the treadmill in 30-minute sessions with 1-minute repetitions and 2-minute active rest between each repetition. This method was also carried out with a gradual increase in the speed. The initial speed was chosen based on the indirect estimation of aerobic power used in the previous studies [22]. In the first week, 1-minute running was performed at a speed of $30-34 \mathrm{~m} / \mathrm{min}$ (90-100\% aerobic power) as well as 2-minute rests running at a speed of $18-22 \mathrm{~m} /$ $\min (50-60 \%$ aerobic power). In the last week, 1-minute running was performed at a speed of $60-66 \mathrm{~m} / \mathrm{min}(90-$ $95 \%$ aerobic power) as well as 2 -minute rests running at a speed of $30-34 \mathrm{~m} / \mathrm{min}$ (50-60\% aerobic power). Each 
session included warm-up (50-60\% aerobic power), core exercise, and cool-down (50-60\% aerobic power) [22].

Aerobic training was carried out with a gradual increase at a speed of $18-22 \mathrm{~m} / \mathrm{min}$ in the first week to $30-34 \mathrm{~m} / \mathrm{min}$ in the final week in the form of running on the treadmill in 30-minute sessions based on the indirect estimation of aerobic power used in previous studies (50-60\% aerobic power) [22].

\section{Biochemical assay}

The rats were sacrificed 48 hours after the last training session. Anesthesia was done by using a combination of xylazine (3-5 mg/kg body weight) and ketamine (30-50 $\mathrm{mg} / \mathrm{kg}$ body weight). The blood samples were immediately discharged into the test tubes containing an anticoagulant EDTA in order to measure the plasma irisin levels. Soleus muscle and abdominal adipose tissue were removed and transferred to the micro-tube and were then placed in the liquid nitrogen to measure the UCP1 and FNDC5 gene expression. The samples were stored in the freezer at a temperature of $-80{ }^{\circ} \mathrm{C}$ and were then transferred to the laboratory.

Serum concentrations of irisin were analysed by ELISA (BioVendor Laboratory Medicine, Brno, Czech Republic) under standard operating procedures. The kit sensitivity for irisin was $0.01 \mathrm{ng} / \mathrm{ml}$. Irisin kit interand intra-assay coefficients of variation were $10 \%$ and $8 \%$, respectively. A special kit was used to perform the RNA extraction (Qiagen, Hilden, Germany: QIAzol ${ }^{\circledR}$ Lysis Reagent). RNA concentrations were measured (Eppendorff, Germany) with a desired purification of 260 to 280 between 1.8 and 2. Creation of cDNA was also performed using a special kit (QuantiTect Reverse Transcription kit: Qiagen). The created cDNA was kept at $-20^{\circ} \mathrm{C}$ to be used for real-time PCR reactions. To measure gene expression, the real time PCR quantitative method was used by Primix syber green II (Applied Biosystems, Step One, USA). Each reaction and their combined experiments (final volume of 20 $\mu \mathrm{L}$ ) (consisting of $1 \mu \mathrm{L}$ cDNA, $1 \mu \mathrm{L}$ primary forward, 1

Table 1: Characteristics related to the specific primers used in the Real-time PCR.

\begin{tabular}{|c|c|c|c|}
\hline FNDC5 & $\begin{array}{l}\text { F: 5'-GAGGTGCTGATCATCGTCGT-3' } \\
\text { R: 5'-GAGCAAGCACTGAAAGGGTTT-3' }\end{array}$ & 92 & NM_001270981.1 \\
\hline UCP1 & $\begin{array}{l}\text { F: 5'-CAATGACCATGTACACCAAGGAA-3' } \\
\text { R: 5'-CTGACCTTCACСАССTCTGT-3' }\end{array}$ & 387 & NM_012682.2 \\
\hline GAPDH & $\begin{array}{l}\text { F: 5'-GACATGCCGCCTGGAGAAAC-3' } \\
\text { R: 5'-AGCCCAGGATGCCСTTTAGT-3' }\end{array}$ & 200 & XM_017592435.1 \\
\hline
\end{tabular}

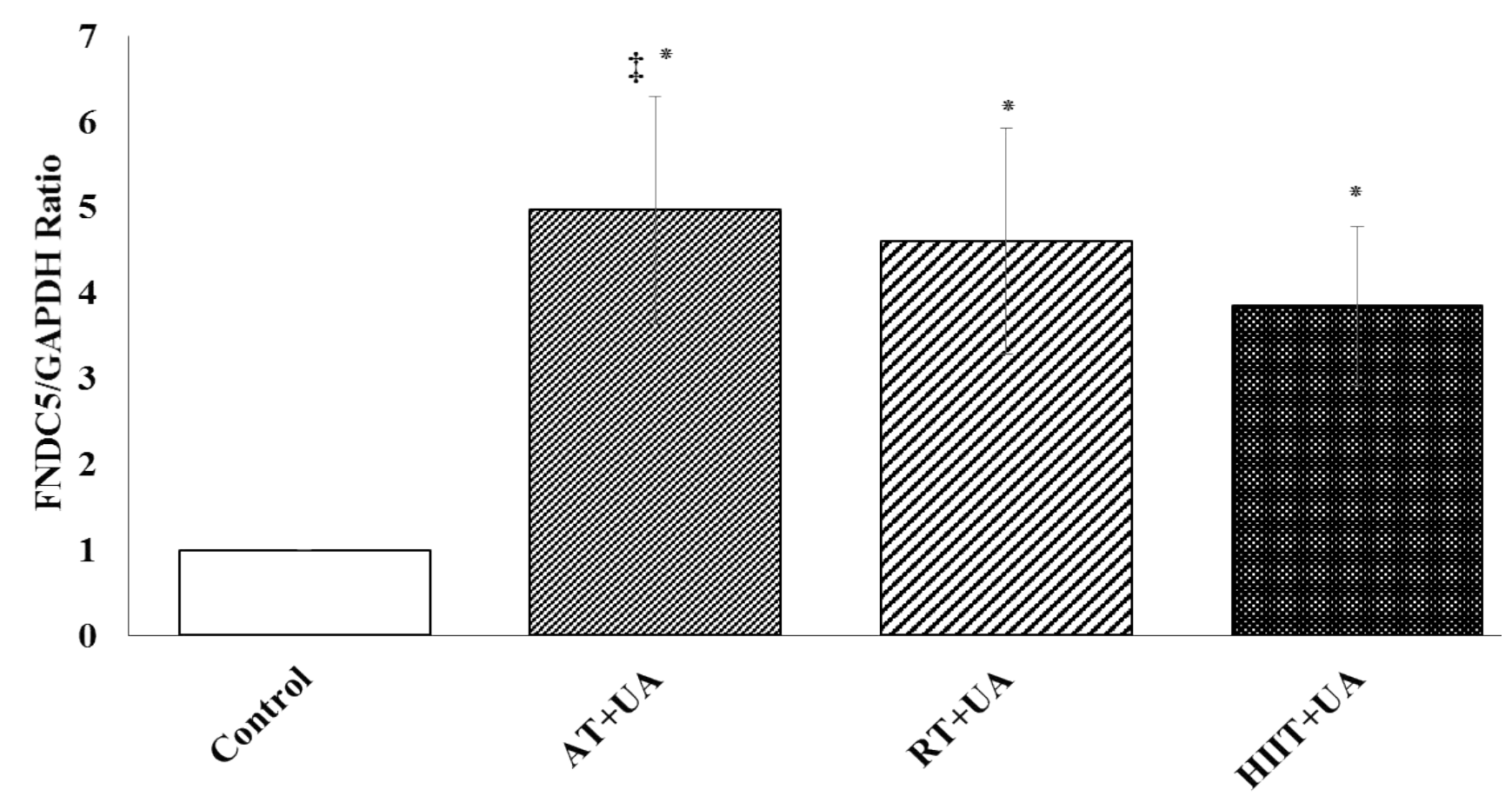

Figure 1: FNDC5 gene expression among the studied groups (means \pm SD). *Represents significant difference compared to the control group. ${ }^{\ddagger}$ Represents significant difference in the AT+UA group compared to the RT+UT and HIIT+UT groups. Control (C), Aerobic Training + Ursolic Acid (AT+UA), Resistance Training + Ursolic Acid (RT+UA), HIIT + Ursolic Acid (HIIT+UA). 
$\mu \mathrm{L}$ reverse primer, $7 \mu \mathrm{L}$ Depc water, and $10 \mu \mathrm{L}$ syber green) were conducted twice (Table 1). Further, GAP$\mathrm{DH}$ was employed as the reference gene. The thermal program used in the real-time PCR consisted of 95 for $10 \mathrm{~min}, 60$ for $15 \mathrm{~s}$, and 72 for $1 \mathrm{~min}$ (replication of 40 cycles). Melt diagram was plotted to investigate the accuracy of the data while standard diagram was drawn to optimize the experimental conditions.

\section{Statistical analysis}

The Shapiro-Wilk test was used to check the normality of the data. Then, the inter-group difference was verified by one-way ANOVA. Bonferroni's post hoc test was used to determine the significance of differences between groups. All statistical methods were performed by SPSS software 22 and at a significance level of $P \leq 0.05$. Excel software 2013 was also used to draw the diagrams.

\section{Results}

There was a significant difference between FNDC5 gene expression in the studied groups $(F=23.444, P=$ $0.001, \eta=0.304)$. The results of Bonferroni's post hoc test showed a higher FNDC5 gene expression in the aerobic training + ursolic acid group $(P=0.01)$ compared with the RT+UT $(P=0.03)$ and HIIT+UT $(P=0.02)$ groups (Figure 1).

In addition, UCP1 gene expression was significantly different in the studied groups $(F=20.315, P=0.001, \eta=$ 0.260). The results of Bonferroni's post hoc test showed a higher mean of UCP1 gene expression in the aerobic training + ursolic acid group $(P=0.01)$ compared to the
$\mathrm{RT}+\mathrm{UT}(\mathrm{P}=0.03)$ and HIIT+UT $(\mathrm{P}=0.02)$ groups (Figure 2).

The concentration of plasma irisin was also significantly different between the studied groups $(P=0.001$, $F=9.207, \eta=0.249)$; this difference was higher in the aerobic training + ursolic acid group $(P=0.01)$ compared to the RT+UT $(P=0.03)$ and HIIT+UT $(P=0.02)$ groups (Figure 3).

\section{Discussion}

The present study showed that administration of 12 weeks of HIIT, resistance and aerobic training along with ursolic acid supplementation is effective on FNDC5 and UCP1 gene expression and plasma irisin levels. The results revealed that aerobic training is more effective than other types of exercise. Muscle tissue FNDC5 and adipose tissue UCP1 and plasma irisin levels were significantly different between the studied groups. FNDC5 and UCP1 gene expression and plasma irisin levels were higher in the aerobic training + ursolic acid group than other groups.

Although, the role of PGC1 $\alpha$-FNDC5-irisin pathway has been studied, it should be noted that in obese subjects, white adipose tissue also secretes irisin and contributes to an increase in the plasma irisin levels, the effect of which is not associated with an increase in the plasma irisin due to the regular physical activity [11]. In addition, changes in the plasma irisin in healthy subjects are dependent on training conditions and exercise mode [12]. There are debates about the effects of the exercise mode on metabol-

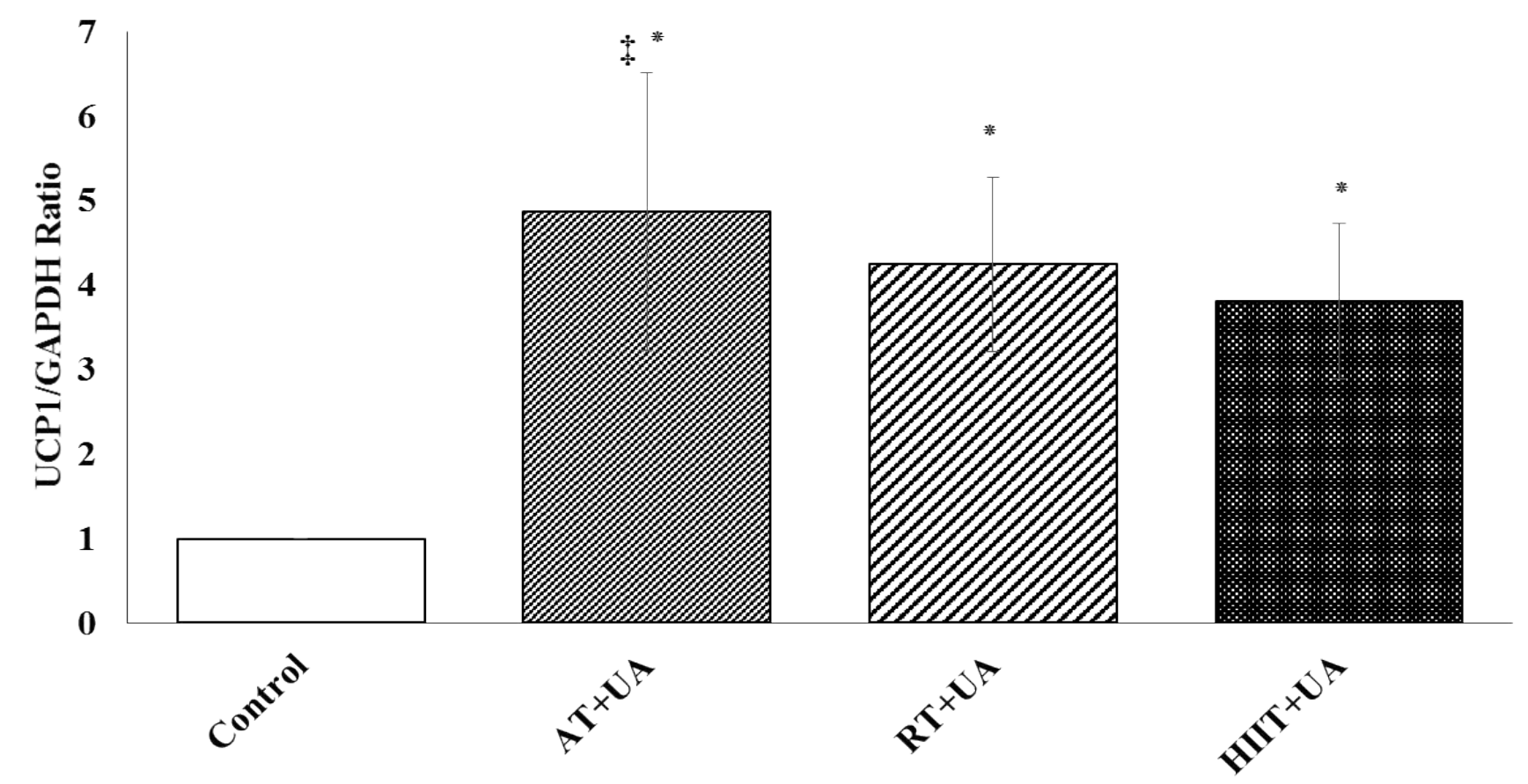

Figure 2: UCP1 gene expression among the studied groups (means $\pm \mathrm{SD}$ ). *Represents significant difference compared to the control group. ${ }^{\ddagger}$ Represents significant difference in the AT+UA group compared to the RT+UT and HIIT+UT groups. Control (C), Aerobic Training + Ursolic Acid (AT+UA), Resistance Training + Ursolic Acid (RT+UA), HIIT + Ursolic Acid (HIIT+UA). 


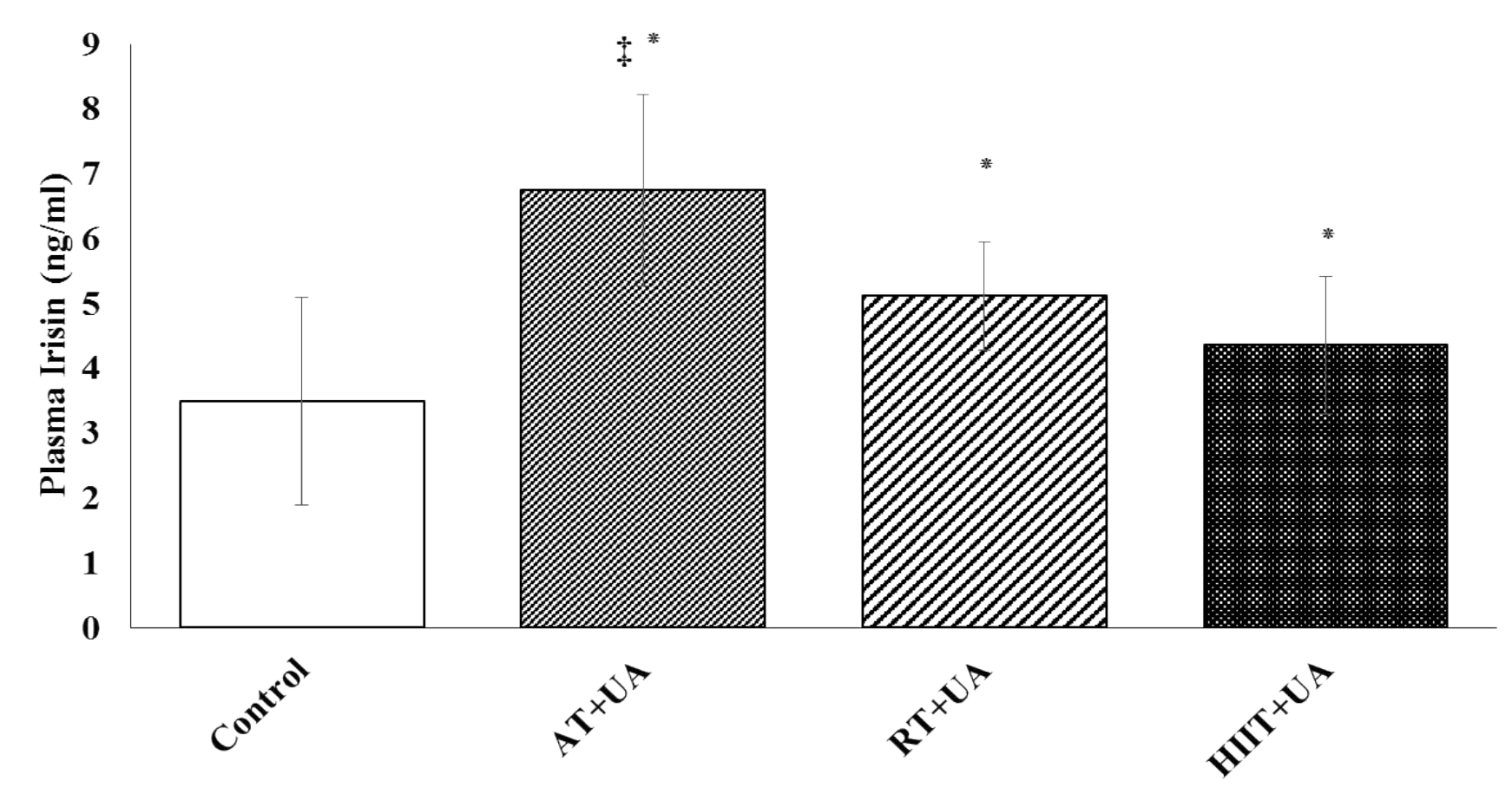

Figure 3: Plasma irisin level among the studied groups (means $\pm \mathrm{SD}$ ). "Represents significant difference compared to the control group. ${ }^{\prime}$ Represents significant difference in the AT+UA group compared to the RT+UT and HIIT+UT groups.

Control (C), Aerobic Training + Ursolic Acid (AT+UA), Resistance Training + Ursolic Acid (RT+UA), HIIT + Ursolic Acid (HIIT+UA).

ic and physiological signaling pathways $[1,23]$. It has been found that, if the intensity and output power of these training modes are identical, they will probably have similar effects $[23,24]$. Exercise training at low intensity is likely to have a greater effective myokines on adipose tissue. It has been shown that, 12-weeks of Nordic walking was effective and increased the plasma irisin levels compared to the resistance training in overweight/obese men with impaired glucose regulation [25].

Concerning the results of the present study, it is important to examine the activity of PGC1 $\alpha$-FNDC5-BDNF axis signaling pathways $[5,6,10]$. This axis by influencing UCP1 can be effective in thermogenesis and the transformation of adipose tissue into Brown Adipose Tissue (BAT). Recently, FNDC5 has been identified as a new actor in metabolic syndrome that, through the mediation of irisin changes the brown adipose tissue $[5,6,10]$. FNDC5 is necessary as an upstream and downstream signaling pathways for metabolism homeostasis, which its lack of adjustment disrupts its metabolic balance. In this signaling pathway, PGC1- $\alpha$ is a major factor [10]. It has been mentioned that, in some tissues including the liver, glucocorticoids receptors are effective in expressing FNDC5 [26]. The effect of this type of receptor can be considered as one of the reasons for better effects of aerobic training observed in the present study. Since, the response of glucocorticoids is delayed, and can be clearly observed in chronic stresses [1].

Like aerobic training, HIIT can be effective in the obesity condition and insulin resistance $[2,3]$. It has been reported that, exercises of HIIT such as decreasing repetitions (involving the decrease in each repetition of the traveled distance) due to maintaining high work intensity have shown better effects [4]. Consequently, one of the reasons for the effects of aerobic training observed in the present study can be the use of HIIT with equal repetitions. Although, it has been shown that, if the level of energy consumption is equal, the irisin response to HIIT would be considered compared to aerobic training [24], the number of the conducted studies is limited and the variety of different training methods does not provide a general conclusion on the effect of HIIT in the metabolic syndrome [3].

Because of the use of fat as a substrate in moderate intensity aerobic training and the involvement of mitochondria in these types of training, it can be said that, possibly irisin influences the adipose tissue and UCP1 stimulation [9]. It is noteworthy that, the changes in the levels of lactic acid were found to be significant, based on the rest periods in HIIT and resistance trainings. In this regard, the levels of the produced lactate can also influence the secretion of growth hormone and testosterone. Hence, the use of high intensity resistance trainings and HIIT may have significant training effects if implemented in healthy subjects. Probably HIIT program as a modality can create a metabolic cycle between skeletal muscle and adipose tissues.

Resistance trainings even in elderly groups have been shown to increase plasma irisin levels, suggesting 
that aging and decreased physical activity concurrently can be among the reasons for changes in the distribution of adipose tissue in the elderly [27]. Study on the simultaneous efficacy of ursolic acid supplementation along with resistance training has shown that, the synchronization of these interventions can increase the levels of plasma irisin [20]. The simultaneous use of ursolic acid and low-intensity trainings on the treadmill has been shown to improve the muscle atrophy in rats [28]. In addition, ursolic acid stimulates the expression of the UCP genome and protects the H9c2 liver cells from hypoxia damage through the p38 signaling pathway [29]. It has been reported that, the effect of ursolic acid supplementation, especially in human liver cells (huh7) is influenced by the PI3K/Akt and MAPK signaling pathway [30]. Note that these are the main pathways to the effect of resistance training, and probably the pattern of resistance training and other interventions such as eccentric contraction should also be considered in future studies.

Regarding the changes observed in present study, aerobic training has a greater effect on the conversion of white-to-brown adipose tissue in rats than other exercise modes (Based on changes of irisin, FNDC5 and UCP1). Studying different physiological responses suggests that, some of these adaptations are more responsive to resistance training, and perhaps some of them are more responsive to HIIT training. Even though, the optimal use of time and reaching the maximum results in the shortest possible time are among the reasons for using HIIT, long duration exercise training at low intensity is likely to have a greater effective myokines on adipose tissue involving the FNDC5/irisin pathway.

Actually, the nature of the studied variable can be effective in the result of the studies, indicating the specificity principle of training. However, in this field, it is difficult to achieve an integrated result due to the use of different studied groups such as animals or healthy human groups and patients. It should also be noted that, the administration of the exercise mode especially the resistance training is very different in terms of repetition and rest time in animals, and therefore, it is difficult to achieve a specific result. The resistance training conducted in human studies was also followed aiming at achieving different goals such as hypertrophy, involving the administration of different exercise modes and speeds. Ultimately, according to the findings of the present study, it is recommended to perform regular physical activity, especially aerobic trainings along with ursolic acid supplementation for the formation of brown adipose tissue as well as considering its optimal use with respect to its metabolic effects on the health.

\section{Disclosure}

The authors of the present study report no conflict of interest.

\section{References}

1. Azarbayjani MA, Fatolahi $H$, Rasaee MJ, Peeri M, Babaei $R$ (2011) The effect of exercise mode and intensity of sub-maximal physical activities on salivary testosterone to cortisol ratio and alpha-amylase in young active males. Int J Exerc Sci 4: 283-293.

2. de Matos MA, Vieira DV, Pinhal KC, Lopes JF, Dias-Peixoto MF, et al. (2018) High-Intensity Interval Training Improves Markers of Oxidative Metabolism in Skeletal Muscle of Individuals With Obesity and Insulin Resistance. Front Physiol 9: 1451 .

3. da Silva DE, Grande AJ, Roever L, Tse G, Liu T, et al. (2019) High-Intensity Interval Training in Patients with Type 2 Diabetes Mellitus: a Systematic Review. Curr Atheroscler Rep 21: 8.

4. Stavrinou PS, Bogdanis GC, Giannaki CD, Terzis G, Hadjicharalambous M (2019) Effects of high intensity interval training frequency on perceptual responses and future physical activity participation. Appl Physiol Nutr Metab 44: 952-957.

5. Bhatt PS, Dhillo WS, Salem V (2017) Human brown adipose tissue-function and therapeutic potential in metabolic disease. Curr Opin Pharmacol 37: 1-9.

6. Bakhshalizadeh S, Rabiee F, Shirazi R, Ghaedi K, Amidi $F$, et al. (2018) Assessment of PGC1alpha-FNDC5 Axis in Granulosa Cells of PCOS Mouse Model. J Reprod Infertil 19: 89-94.

7. Bostrom $P, W u$ J, Jedrychowski MP, Korde A, Ye L, et al. (2012) A PGC1-alpha-dependent myokine that drives brown-fat-like development of white fat and thermogenesis. Nature 481: 463-468.

8. Fenzl A, Kiefer FW (2014) Brown adipose tissue and thermogenesis. Horm Mol Biol Clin Investig 19: 25-37.

9. Otero-Díaz B, Rodríguez-Flores $M$, Sánchez-Muñoz V, Monraz-Preciado F, Ordoñez-Ortega S, et al. (2018) Exercise Induces White Adipose Tissue Browning Across the Weight Spectrum in Humans. Front Physiol 9: 1781.

10. Dinas PC, Lahart IM, Timmons JA, Svensson PA, Koutedakis Y, et al. (2017) Effects of physical activity on the link between PGC-1a and FNDC5 in muscle, circulating lotarisin and UCP1 of white adipocytes in humans: A systematic review. F1000Research 6: 286.

11. Pardo $M$, Crujeiras $A B$, Amil M, Aguera Z, Jimenez-Murcia $S$, et al. (2014) Association of irisin with fat mass, resting energy expenditure, and daily activity in conditions of extreme body mass index. Int J Endocrinol 2014: 857270.

12. Quu S, Bosnyak E, Treff G, Steinacker JM, Niess AM, et al. (2018) Acute exercise-induced irisin release in healthy adults: Associations with training status and exercise mode. Eur J Sport Sci 18: 1226-1233.

13. Katashima CK, Silva VR, Gomes TL, Pichard C, Pimentel GD (2017) Ursolic acid and mechanisms of actions on adipose and muscle tissue: a systematic review. Obes 18: 700-711.

14. Skinner RC, Gigliotti JC, Ku KM, Tou JC (2018) A comprehensive analysis of the composition, health benefits, and safety of apple pomace. Nutrition Reviews 76: 893-909.

15. Alam P, Al-Yousef HM, Siddiqui NA, Alhowiriny TA, Alqasoumi SI, et al. (2018) Anticancer activity and concurrent analysis of ursolic acid, $\beta$-sitosterol and lupeol in three different Hibiscus species (aerial parts) by validated HPTLC method. Saudi Pharmaceutical Journal 26: 1060-1067. 
16. Seo DY, Lee SR, Heo JW, No MH, Rhee BD, et al. (2018) Ursolic acid in health and disease. Korean J Physiol Pharmacol 22: 235-248.

17. Nguyen HN, Ahn YJ, Medina EA, Asmis R (2018) Dietary 23-hydroxy ursolic acid protects against atherosclerosis and obesity by preventing dyslipidemia-induced monocyte priming and dysfunction. Atherosclerosis 275: 333-341.

18. Jia Y, Kim S, Kim J, Kim B, Wu C, et al. (2015) Ursolic acid improves lipid and glucose metabolism in high-fat-fed C57BL/6J mice by activating peroxisome proliferator-activated receptor alpha and hepatic autophagy. Mol Nutr Food Res 59: 344-354.

19. Kaji H (2018) [Body weight and bone/calcium metabolism. Muscle, myokines and bone/calcium metabolism.]. Clin Calcium 28: 919-926.

20. Bang HS, Seo DY, Chung YM, Kim DH, Lee SJ, et al. (2017) Ursolic acid supplementation decreases markers of skeletal muscle damage during resistance training in resistance-trained men: a pilot study. Korean J Physiol Pharmacol 21: 651-656

21. Hornberger TA, Jr, Farrar RP (2004) Physiological hypertrophy of the FHL muscle following 8 weeks of progressive resistance exercise in the rat. Can J Appl Physiol 29: 16-31.

22. Rahmati-Ahmadabad $S$, Azarbayjani MA, Farzanegi $P$, Moradi $L$ (2019) High-intensity interval training has a greater effect on reverse cholesterol transport elements compared with moderate-intensity continuous training in obese male rats. Eur J Prev Cardiol 12.

23. Castro A, Duft RG, Ferreira MLV, Andrade ALL, Gaspari AF, et al. (2019) Association of skeletal muscle and serum metabolites with maximum power output gains in response to continuous endurance or high-intensity interval training programs: The TIMES study - A randomized controlled trial. PLoS One 14: e0212115.

24. Tsuchiya $Y$, Ando D, Goto K, Kiuchi M, Yamakita M, et al. (2014) High-intensity exercise causes greater irisin response compared with low-intensity exercise under similar energy consumption. Tohoku J Exp Med 233: 135-140.

25. Korkmaz A, Venojarvi M, Wasenius $N$, Manderoos $S$, Deruisseau KC, et al. (2019) Plasma irisin is increased following 12 weeks of Nordic walking and associates with glucose homoeostasis in overweight/obese men with impaired glucose regulation. Eur J Sport Sci 19: 258-266.

26. Kim HK, Jeong YJ, Song IS, Noh YH, Seo KW, et al. (2017) Glucocorticoid receptor positively regulates transcription of FNDC5 in the liver. Sci Rep 7: 43296.

27. Zhao J, Su Z, Qu C, Dong Y (2017) Effects of 12 Weeks Resistance Training on Serum Irisin in Older Male Adults. Front Physiol 8: 171.

28. Kim JC, Kang YS, Noh EB, Seo BW, Seo DY, et al. (2018) Concurrent treatment with ursolic acid and low-intensity treadmill exercise improves muscle atrophy and related outcomes in rats. Korean J Physiol Pharmacol 22: 427-436.

29. Chen M, Wang X, Hu BO, Zhou J, Wang X, et al. (2018) Ursolic acid stimulates UCP2 expression and protects $\mathrm{H} 9 \mathrm{c} 2$ cells from hypoxia-reoxygenation injury via p38 signaling. $J$ Biosci 43: 857-865.

30. Lee KC, Chen YL, Lin PY, Chuang WL (2018) Ursolic Acid-Induced Apoptosis via Regulation of the PI3K/Akt and MAPK Signaling Pathways in Huh-7 Cells. Molecules 23. 